Roel M. Willems

\title{
Can literary studies contribute to cognitive neuroscience?
}

Keywords: Cross fertilization, Experimental control, Humanities, Interdisciplinary, Neuroscience

Roel M. Willems: The Donders Institute for Brain, Cognition and Behaviour, Radboud University Nijmegen, The Netherlands, and the Max Planck Institute for Psycholinguistics, Nijmegen, The Netherlands. E-mail: r.willems@fcdonders.ru.nl

\section{Introduction}

'Why are your experiments so boring? I almost fell asleep.' This was the honest question and complaint of one of our participants after taking part in an experiment. She had just been engaged in a lexical decision task for a little over an hour. In lexical decision you ask the participant to press one of two buttons as quickly and as accurately as possible to decide whether a letter string is a real word or not. So you see

'retkop'

and you press the button for 'no'. When you see

'rash'

you press the button for 'yes'.

I had to think of this episode after reading the four contributions to this special issue for the Journal of Literary Semantics. Let me explain why.

\section{Experimental control: the craftsmanship of cognitive neuroscientists}

Psychologists (and here I'll focus on cognitive neuroscientists) are masters in experimental design: designing the experiment in such a way that the process under study can be neatly isolated. So all words in our experiment were on 
average of equal length, the non-words did not violate the phonotactic rules of the language we were testing, sometimes participants pressed the left button for 'yes' and sometimes the right, and many more crucial details that I will not bother you with. This is an important skill which is often thought of too lightly; how good one is at experimental design is an important factor in determining the craftsmanship of the experimenter.

The lexical decision task is a classical task for studying word comprehension (or 'word processing'). When we measure neural signals by means of fMRI while people perform this task, and compare neural activation in response to reading words versus to reading non-words, part of the left temporal cortex is found to be activated, and our conclusion will be that temporal cortex is involved in the comprehension of words. Most discussion in experiments like this centres on whether you did the experiment 'right': were all confounding variables controlled for, or is there another, alternative explanation for the results? This is what you will argue about with your reviewers and readers.

One level up there is a more pressing issue, which every cognitive scientist has to face: are we studying what we think we are studying? Am I really a student of the neurobiology of language when I look at brain patterns generated as participants see letter strings flashed on a screen and are instructed to press a button? This is the deeper message behind our participant's question: what does this boring task have to do with language? Our participant probably doesn't find language boring; most people enjoy reading, talking and listening. But then, in real life people are never faced with single words presented outside of a wider context, and asked to press buttons in response.

The reason for the boring tasks is obvious: experimental control. The standard reply goes like this: 'It's simply impossible to study natural language use in the laboratory; there would be so many confounding factors that the results would be uninterpretable. Instead we strip the process under study down to its bare essentials, and measure the subcomponents step-by-step and one at a time.'

We're left with an uneasy feeling: of course, understanding words is part of language comprehension, and of course, watching alternating black-and-white rectangles can be called 'visual perception'. But it's far from the sensation I have when typing this piece, or when looking around my office. It feels like we've thrown out the baby with all the confounding variables. This is where I see the great opportunity for cross-talk between (literary) humanities and cognitive neuroscience. The four contributions to this special issue stay close to what the comprehension of literature is like in reality, and at the same time remain conceptually rigorous and take the finesses of language comprehension seriously. This is the sort of craftsmanship that can nicely complement the cognitive neuroscientist's skills. 


\section{The contributions to this special issue}

In this special issue, four scholars from the literary humanities explore how their expertise can feed into cognitive (neuro)science. This is a fresh perspective, since the issue is more often phrased the other way around: how can cognitive neuroscience be used to test hypotheses from other disciplines such as the humanities? I will briefly discuss what I got out of the four contributions, but before I do so I want to stress that I much enjoyed reading them. I hope I characterise the articles rightfully below, and I apologise in advance if I miss the point in my short descriptions below, or otherwise misinterpret the authors' ideas. It is my intention not to provide a full overview of the papers, but rather to give a global impression.

Hogan discusses how James Joyce expresses the parallel nature of thought in a serial medium, namely written text. Several processes happen simultaneously (in parallel) in thought; the mind does not keep itself busy with one thing at a time. This parallelism is, however, easily missed in an introspective description of thought, as in a novel. We tend to describe our thoughts in a linguistic format, in which things happen serially, one by one. By showing that Joyce chooses to describe thinking as parallel in parts of his novel, Hogan raises awareness of the parallelism of thinking.

Polvinen explores the relationship between the emotional engagement of readers with a text on the one hand, and the self-reflective commentary of the writer or storyteller on the other. One position is that such self-reflective comments disengage the reader from the text, thereby distracting him or her from the main story. Quite to the contrary, Polvinen argues that self-reflection should be considered a form of joint attention between the reader and the storyteller, in which the main story of the text is the object of shared attention. Instead of distracting a reader, it is suggested that by exploiting an important mechanism of social cognition (joint attention), the writer engages readers' emotions more strongly than would be possible if the text contained no such self-reflective elements.

Troscianko discusses the role of imagery in literary reading, with reference to the so-called imagery debate in cognitive science. She defends an enactive view on imagery, inspired by enactive views on perception (O'Regan and Noë 2001). The main thesis is that vision (and imagery) is a mode of exploration of the world. Perceiving the colour or shape of an object, for instance, can be understood as mediated by the relevant set of the sensorimotor contingencies that we have learned over the course of development. For instance, we know how the image would change should we move our eyes or body with respect to the object, and it is this (implicit) sensing that constitutes seeing. An analogue is finding out what object is in front of you (e.g. a bottle) when closing your eyes and only exploring 
the object with your fingers (O’Regan and Noë 2001: 945). When doing this, it is clear that there will be 'holes' in your perception: your fingers cannot contain the bottle fully at any given moment, and the same may be true for visual perception and imagery. Troscianko's contribution nicely makes the case for noncommittal imagery being abundant in literary texts, while at the same time being actively ignored in questionnaires used to test imagery abilities. She goes on to show data that reveal a mismatch in some individuals between imagery abilities as assessed with standard psychological measures (questionnaires), and their report of imagery during reading of literary texts.

Finally, Burke asks whether findings in cognitive (neuro)science regarding storage and retrieval of words and images can be extended to aesthetic objects, such as the style of a text. He goes on to discuss several kinds of style motifs in a writer's repertoire, such as chiasmus. Writers use these style figures because they work: they engage the reader successfully (and often unconsciously) with the text being read. Why are these style figures effective? The hypothesis is that they are part of a cultural heritage which is reflected in the makeup of the brain. That is, style figures work because they stimulate those things in the human mind that humans like. They probably make use of evolutionarily ancient structures, an example of recycling a neural area's function for another purpose. The challenge will be to find out what 'those things' in the brain are: why do we enjoy metre, what is it that makes style figures work in neuroscientific terms? In other words, what is the neural target domain to which metre is mapped?

\section{Can literature studies illuminate cognitive neuroscience?}

Now back to the main question: can these insights illuminate cognitive neuroscience? I do think that this is the case, and that there is room for cross-fertilisation, and benefits to be gained from it. Broadly speaking, the main lesson for cognitive neuroscience of language, from these (and other) contributions from the humanities, is to stay much closer to actual language use. Here is where I link back to the first part of my piece: the decontextualised style of researching language, with its laboratory-created stimuli and tasks. All the contributions in this special issue study phenomena that are much richer in cognitive terms than what is often the topic of study in current cognitive neuroscience of language.

But there was a reason for using the simplistic tasks that we talked about: experimental control. Don't we by definition give up experimental control when increasing the psychological reality of our experiments? This is a rightful fear 
for experimentalists, and most stop there. It is encouraging, however, to see that more and more researchers are trying out and developing new technologies for data analysis of less constrained language stimuli. One example methodology is inter-participant correlations, in which the time course of the fMRI BOLD signal is correlated between participants, in order to identify brain areas that are responsive to a story or movie in a similar way across participants (e.g. Kauppi et al. 2010, Lerner et al. 2011, Nummenmaa et al. 2012, Wilson et al. 2008). It is still early days, and the full potential of these new developments has not been explored yet, but the important message is that modern techniques allow one to go beyond the traditional time-lock-and-average style of data analysis, freeing up room for more naturalistic stimuli to be used while retaining the necessary amount of experimental control. This is not to say that it is easy to do. There are serious constraints when measuring people's brain activity: participants often have to sit still and are in confined and isolated rooms, and some methods make a whole lot of noise. But the developments described here may make it possible to integrate cognitive neuroscience more productively with literary study than has hitherto been the case.

Another area where I see fertile ground for collaboration is conceptual rigour and finesse in terminology. For example, part of my work has been on sensorimotor simulation during language comprehension, that is, the activation of sensorimotor cortices when reading or listening to language. In most of these papers, you will in vain look for a definition of simulation. Moreover, we treat simulation as a one-of-a-kind phenomenon, as if all simulation is the same (but see Willems et al. 2010). The Troscianko paper in this issue nicely provides a taxonomy for imagery during language comprehension, and provides the necessary conceptual vocabulary that we (I) have ignored in my experimental work.

In conclusion, I feel that the literary humanities can influence cognitive neuroscience and that there are definitely opportunities for fertile collaboration between these academic fields. Time will tell how exactly this will work, and in the meantime we'd probably best get started. The promise is that the psychological reality of our work will increase, that we will forge a more obvious link with real behaviour, and - a welcome extra - that we might even end up with slightly less bored experimental participants.

\section{References}

Kauppi, Jukka-Pekka, Jääskeläinen, liro P., Sams, Mikko, and Tohka, Jussi (2010). Inter-subject correlation of brain hemodynamic responses during watching a movie: localization in space and frequency. Frontiers in Neuroinformatics 4(5). doi:10.3389/fninf.2010.00005. 
Lerner, Yulia, Honey, Christopher J., Silbert, Lauren J., and Hasson, Uri (2011). Topographic mapping of a hierarchy of temporal receptive windows using a narrated story. The Journal of Neuroscience 31: 2906-15. doi:10.1523/JNEUROSCI.3684-10.2011.

Nummenmaa, Lauri, Glerean, Enrico, Viinikainen, Mikko, Jääskeläinen, liro P., Hari, Riitta, and Sams, Mikko (2012). Emotions promote social interaction by synchronizing brain activity across individuals. Proceedings of the National Academy of Sciences of the United States of America 109: 9599-604. doi:10.1073/pnas.1206095109.

O’Regan, J. Kevin, and Noë, Alva (2001). A sensorimotor account of vision and visual consciousness. Behavioral and Brain Sciences 24: 939-73.

Willems, Roel M., Toni, Ivan, Hagoort, Peter, and Casasanto, Daniel (2010). Neural dissociations between action verb understanding and motor imagery. Journal of Cognitive Neuroscience 22: 2387-400.

Wilson, Stephen M., Molnar-Szakacs, Istvan, and Iacoboni, Marco (2008). Beyond superior temporal cortex: intersubject correlations in narrative speech comprehension. Cerebral Cortex 18: 230-42. doi:10.1093/cercor/bhm049.

\section{Bionote}

Roel M. Willems obtained his $\mathrm{PhD}$ from Radboud University Nijmegen on a thesis investigating how information from spoken language and from co-speech gestures is integrated in the human brain. He later investigated the involvement of sensorimotor cortices in language understanding, using methodologies like fMRI, EEG, and TMS. After a postdoctoral visit to UC Berkeley, he is now at the Donders Institute for Brain, Cognition and Behaviour and the Max Planck Institute for Psycholinguistics, both in Nijmegen, The Netherlands. His current research uses neuroimaging methods to gain insight into simulation during narrative comprehension. 